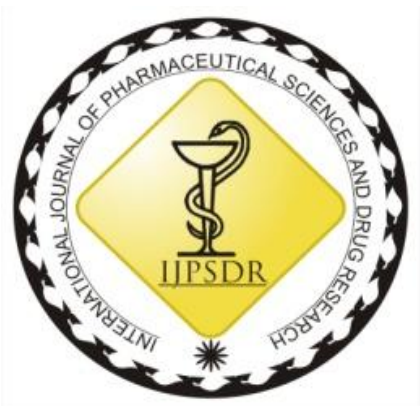

ISSN: 0975-248X

RESEARCH ARTICLE CODEN (USA): IJPSPP $(\mathrm{cc})$ EY-NG-SA

\title{
In vivo Evaluation of Tazarotene Solid Lipid Nanoparticles Gel For Topical Delivery
}

\author{
Rajkumar Aland ${ }^{*}$, M. Ganesan², P. Rajeswara Rao ${ }^{3}$ \\ ${ }_{1}^{1}$ Jawaharlal Nehru Technological University, Kakinada-533003, Andhra Pradesh, India \\ ${ }^{2}$ Dr. Reddy's Laboratories Limited, Hyderabad-502325, Telangana, India \\ ${ }^{3}$ Andhra University, Visakhapatnam-530003, Andhra Pradesh, India
}

Copyright (C) 2019 Rajkumar Aland et al. This is an open access article distributed under the terms of the Creative Commons AttributionNonCommercial-ShareAlike 4.0 International License which allows others to remix, tweak, and build upon the work non-commercially, as long as the author is credited and the new creations are licensed under the identical terms.

\begin{abstract}
The purpose of this research work was to develop and optimize the Solid Lipid Nanoparticles (SLNs) of Tazarotene for the effective topical delivery in the treatment of psoriasis. Tazarotene loaded SLNs were prepared by hot homogenization followed by the ultrasonication using Taguchi's design and based on the results further investigation was made using central composite design. The lipid Dynasan-116, surfactant poloxomer-188 and co surfactant egg lecithin resulted in better percent drug loading and evaluated for particle size, zeta potential, TEM, drug entrapment efficiency, in vitro drug release and stability. All parameters were found to be in an acceptable range. In vitro drug release of optimized SLN formulation (F1) was found to be $98.12 \pm 1.52 \%$, whereas pure drug release was 42.12 after $60 \mathrm{~min}$. The optimized formulation was incorporated into the gel. The release rate (flux) of tazarotene across the membrane and excised skin differs significantly. The accumulative amount of Tazarotene in skin from SLN based gel formulation and marketed gel were $41.12 \pm 0.12 \mathrm{mg}$ and $30.02 \pm 0.04$ mg respectively. This result supported our hypothesis made in skin permeation studies on rat skin. From histopathological studies the microscopic observations indicate that the optimized SLN formulation, SLN based gel formulation and marketed gel has no significant effect on the microscopic structure of the skin. The skin-irritation studies indicated that SLN based gel containing Tazarotene did not show any sign of skin irritation as compared to moderate erythema shown by marketed gel formulation (Tazret@ gel) after $72 \mathrm{~h}$ of application. Thus, SLN based gel formulation demonstrated advantage over marketed formulation in improving the skin tolerability of Tazarotene indicating their potential in improving patient acceptance and topical delivery of Tazarotene.
\end{abstract}

Keywords: Tazarotene, Psoriasis, Topical gel, Skin irritation studies, Histopathological studies.

\section{INTRODUCTION}

Topical drug delivery is one of the most exciting and challenging issues for the pharmaceutical scientist. ${ }^{[1-2]}$
These lipid nanoparticles have the potential to increase cutaneous drug delivery of both hydrophilic and lipophilic drugs when compared to the conventional 
formulations. [3-4] Topical application of Solid lipid nanoparticles (SLNs) and nanostructured lipid carriers (NLCs) have been developed as novel systems that are composed of physiological lipid materials suitable for topical, dermal and transdermal administration. [5] Tazarotene, is 6-[2-(4,4-dimethylthiochroman- 6yl)ethynyl] nicotinic acid ethyl ester, a member of a new generation of receptor selective synthetic retinoids, is used for the topical treatment of mild to moderate plaque psoriasis, acne vulgaris, and photoaging. The conventional formulations of Tazarotene (gel, cream, aerosol, and foam) could be less efficacious due to insignificant skin penetration properties. Improving the permeation of some agents to the dermis layer of skin is desired in the form of gel deeply through the epidermal layers. [6]

Thus, topical application of SLNs based gel with increased penetration and retention through skin because of lipid nanoformulation will be much promising for the topical treatment of psoriasis. The aim of the present work was to explore the potential benefits of SLNs in improving the topical delivery of Tazarotene. The formulation was optimized by using 3factor, 3-level Central composite design. The optimized SLN preparation of acitretin was incorporated in gel and the gel preparation was evaluated for in vitro skin permeation study, skin retention, histopathological investigation of skin and skin-irritation testing.

\section{MATERIALS AND METHODS}

\section{Materials}

Tazarotene was obtained as a gift sample from Dr. Reddy's Laboratory Ltd., Hyderabad, India. Trimyristin (Dynasan-114), tripalmitin (Dynasan-116) and tristearin (Dynasan-118) were purchased from Sigma-Aldrich Chemicals, Hyderabad, India. Egg Lecithin, Carbopol and Poloxamer-188 were gift samples from Aurobindo Labs, India. All other chemicals and solvents were of analytical grade and were used without further purification.

Dialysis Bag (Molecular weight cut off $10 \mathrm{kDa}$ ) was purchased from Hi-media Pvt. Ltd., Trehalose dehydrate was purchased from Sigma Chemicals Co. All other chemicals used were of analytical grade or spectroscopic grade.

Preparation of Tazarotene loaded solid lipid nanoparticles

Tazarotene loaded SLNs were prepared by hot homogenization followed by the ultrasonication. ${ }^{[7-8]}$ Tazarotene, lipid and egg lecithin were dissolved in 5 $\mathrm{ml}$ of 1:1 mixture of chloroform and methanol. Organic solvents were completely removed using a rota evaporator. The drug embedded lipid layer was molten by heating to $5^{\circ} \mathrm{C}$ above melting point of the lipid. Aqueous phase was prepared by dissolving Poloxamer 188 in double distilled water and heated to same temperature (based on lipid melting point) of oil phase. Hot aqueous phase was added to the oil phase, and homogenization was carried out (at $12000 \mathrm{rpm}$ ) using homogenizer for $4 \mathrm{~min}$. The coarse hot oil in water emulsion so obtained was ultrasonicated using a $12 \mathrm{~T}$ probe Sonicator for $20 \mathrm{~min}$. Tazarotene loaded solid lipid nanoparticles were obtained by allowing hot nanoemulsion to cool to room temperature.

Characterization of Tazarotene loaded solid lipid nanoparticles

The mean particle size, polydispersity index and zeta potential of Tazarotene nanoparticles were determined by laser light diffractometry using Zetasizer nanoseries, SM2000K (Malvern Instruments Inc., UK). Lyophilization of SLNs conducted at $-40^{\circ} \mathrm{C}$ for overnight. ${ }^{[9]}$ Entrapment efficiency was determined by measuring the concentration of unentrapped free drug in aqueous medium containing either PVA or PLX, percentage drug loading studied by UV absorbance at $246 \mathrm{~nm}$. [10] The Tazarotene, Dynasan-116, poloxomer188, egg lecithin and physical mixtures (PM in 1:1 ratio), and lyophilized Tazarotene nanoparticles were further characterized by DSC.

\section{Design of experiments}

Conventional statistical experiment design determine the optimal conditions on the basis of the measured values of the characteristic properties, while Taguchi method determine the experimental conditions having the variability as the optimum conditions. [11] 18 experiments with one 2-level factor and seven 3-level factors were conducted. The Stat-Ease Design Expert ${ }^{\circledR}$ software V8.0.1 was used for regression and graphical analysis of the obtained data. For our experiments, we considered eight parameters (Surfactant type, Type of lipid, Surfactant concentration, Co-surfactant concentration, Lipid-to-drug ratio, ChloroformMethanol ratio, Organic-aqueous phase ratio and sonication time). Taguchi's orthogonal array is chosen with eight parameters that could affect the particle size and entrapment efficiency.

\section{Preparation of Tazarotene loaded SLN gel}

The Tazarotene loaded SLN gel formulation was prepared by dispersing required quantity of Carbopol $934 \mathrm{P}(1 \% \mathrm{w} / \mathrm{w})$ in small quantity of distilled water and allowing to hydrate for $4-5$ h. ${ }^{[12]}$ Propylene glycol $(10 \%$ $\mathrm{w} / \mathrm{w})$ and glycerol $(30 \% \mathrm{w} / \mathrm{w})$ were subsequently added to the aqueous dispersion. Inclusion of air was avoided by addition of $0.5 \mathrm{ml}$ of triethanolamine and lyophilized Tazarotene nanoparticles powder equivalent to $150 \mathrm{mg}$ of the drug was incorporated into the gelunder gentle stirring. Finally, rest of the water $(58.85 \% \mathrm{w} / \mathrm{w})$ was added to make up the volume of aqueous dispersion up to $100 \% \mathrm{w} / \mathrm{w}$.

\section{Characterization of Tazarotene SLN based gel}

The SLN based gel of Tazarotene was characterized by determination of drug content by UV Spectroscopy, determination of spreadability and $\mathrm{pH}$ of SLN gel. [13]

In vitro drug release studies

In vitro release studies were performed using dialysis bag method with PBS (pH 7.4) as dissolution medium at $37^{\circ} \mathrm{C}$ with the speed of $50 \mathrm{rpm}$. At predetermined time points, $3 \mathrm{ml}$ of dissolution medium was removed 
and filtered as described above and analyzed by UVVisible spectrophotometer at $246 \mathrm{~nm}$. The removed volume was replaced with the same volume of a PBS.

Rheological studies on the micro emulsion based gel Brookfield Viscometer LVDV - IIIU (Brookfield Engineering LABS, Stoughton, USA) with helipath stand was used for rheological studies. $30 \mathrm{~g}$ of sample was placed in a beaker and was allowed to equilibrate for $5 \mathrm{~min}$ before measuring the dial reading using a T-C spindle at $0.5,1,2.5$, and $5 \mathrm{rpm}$. ${ }^{[14]}$ The measurements were carried in triplicate at room temperature

\section{In-vitro Skin Permeation Study}

The in vitro skin permeation study was carried out as per the Committee for the Purpose of Control and Supervision of Experiments on Animal (CPCSEA) guidelines. The study protocols were approved by Institutional Animal Ethics Committee (IAEC) with No. 1292/ac/09/CPCSEA-18/A. The abdominal skins obtained from male Wistar rats weighing $250 \pm 10 \mathrm{~g}$ (68 weeks) were used for in vitro permeation experiments of prepared formulations. After hair was shaved carefully with an electric clipper, the skin was excised from the abdominal region of each sacrificed rat and the subcutaneous fat and other extraneous tissues were removed without damaging the epidermal surface. The excised rat skin samples of almost the same thickness were rinsed with physiological saline and placed in refrigerator at $4^{\circ} \mathrm{C}$ prior to permeation experiments. [1516] The skin membranes were first hydrated for $30 \mathrm{~min}$ in buffer solution ( $\mathrm{pH}$ 7.4) at room temperature to remove extraneous debris and leachable enzymes.

The rat skin was mounted between the donor and receptor compartment with the stratum corneum facing upper side on the Franz diffusion cell. To maintain sink conditions $30 \%(\mathrm{v} / \mathrm{v})$ ethanol in phosphate buffer $\mathrm{pH}$ 7.4 was taken in receptor compartment. The temperature was maintained at $37 \pm 1^{\circ} \mathrm{C}$. Diffusion media was continuously stirred with Teflon coated magnetic bar at a constant rate, in a way that the rat skin surface just flushes the diffusion fluid. The formulation $(1 \mathrm{~g})$ was gently placed in a donor chamber and then the diffusion cells were covered with an aluminum foil to prevent light exposure. At 1, 2, 4, 6, 8, 10 , and $12 \mathrm{~h}$ aliquots of $1 \mathrm{ml}$ sample were withdrawn from the receptor compartment and replaced immediately with an equivalent volume of receptor fluid. The samples were analysed for drug content using UV spectrophotometer at $246 \mathrm{~nm}$. Each experiment was performed in triplicate. The cumulative amount of drug permeated (Q) at different time intervals and various parameters like steady state flux $\left(\mathrm{J}_{\mathrm{ss}}\right)$, permeability coefficient $\left(\mathrm{K}_{\mathrm{p}}\right)$ and enhancement ratio (ER) were calculated .

\section{Skin retention study}

Skin retention study was performed in order to analyze the content of the drug in the skin. At the end of the in vitro skin permeation study, the skin samples were washed with water and methanol on both sides and carefully dried. [17] Then a defined amount of methanol was added to each piece of skin. The samples were vortexed for $10 \mathrm{~min}$ in order to extract its drug content and stirred overnight. After centrifugation the samples were analyzed by UV spectrophotometer.

Histopathological investigation of skin

The rat abdominal skin was mounted on the Franz diffusion cell. The optimized gel formulation, and marketed gel were applied similar to the method of permeation study and the effects were compared against control. [18] A piece of fresh excised untreated skin sample was used as control. The skin was fixed in $10 \%$ neutral formalin for $24 \mathrm{~h}$ and then cut vertically against the surface at the central region (4 mm width). Each section was dehydrated using graded solutions of ethanol and then embedded in paraffin wax. Tissues were divided into small pieces and stained with hematoxylin and eosin. The sections were observed under 100x magnifications and photographed.

Skin-irritation testing (Draize patch test)

The irritation potential of the SLN based Tazarotene gel in comparison with marketed Tazarotene gel was evaluated by carrying out the Draize patch test on rabbits. [19] Animal care and handling throughout the experimental procedure were performed in accordance with the CPCSEA guidelines. The experimental protocol was approved by the institutional Animal Ethical Committee (IAEC). White New Zealand rabbits weighing $2.5-3 \mathrm{Kg}$ were acclimatized before the beginning of the study

Animals were divided into four groups $(n=3)$ as follows:

Group 1: No application (control).

Group 2: Marketed formulation (Tazarotene gel containing $0.05 \% \mathrm{w} / \mathrm{w})$.

Group 3: Gel formulation without Tazarotene (placebo gel).

Group 4: SLN based gel containing Tazarotene $(0.05 \%$, $\mathrm{w} / \mathrm{w})$.

The back of the rabbits were clipped free of hair $24 \mathrm{~h}$ prior to the application of the formulations. $0.5 \mathrm{~g}$ formulations were applied on the hair free skin of rabbits by uniform spreading within the area of $4 \mathrm{~cm}^{2}$. The skin was observed for any visible change such as erythema (redness) at 24, 48 and $72 \mathrm{~h}$ after the application of various formulations. The mean erythemal scores were recorded (ranging from 0 to 4 ) depending on the degree of erythema as follows: no erythema $=0$, slight erythema (barely perceptible-light pink $)=1$, moderate erythema (dark pink) $=2$, moderate to severe erythema (light red) $=3$, and severe erythema (extreme redness) $=4$.

\section{RESULTS AND DISCUSSION}

\section{Optimization and confirmation experiment}

All the prepared formulations (Table 1) were analyzed in order to determine their particle size distribution, PDI and zeta potential. 
Table 1: Experimental batches (Taguchi orthogonal array table of L-18)

\begin{tabular}{|c|c|c|c|c|c|c|c|c|c|c|c|c|c|c|c|c|c|}
\hline \multirow[b]{2}{*}{$\begin{array}{c}\text { Expt. } \\
\text { No. }\end{array}$} & \multicolumn{8}{|c|}{ Experimental conditions } & \multicolumn{3}{|c|}{ Particle size (nm) } & \multicolumn{3}{|c|}{ Entrapment efficiency (\%) } & \multicolumn{3}{|c|}{ \% Drug loading } \\
\hline & A & B & $\mathrm{C}$ & D & $\mathbf{F}$ & G & $\mathbf{H}$ & I & $\begin{array}{c}\text { Actual } \\
\text { value }\end{array}$ & $\begin{array}{l}\text { Predicted } \\
\text { value }\end{array}$ & $\begin{array}{c}\mathrm{S} / \mathrm{N} \\
\text { ratio }\end{array}$ & $\begin{array}{c}\text { Actual } \\
\text { value }\end{array}$ & $\begin{array}{c}\text { Predicted } \\
\text { value }\end{array}$ & $\begin{array}{c}\mathrm{S} / \mathrm{N} \\
\text { ratio }\end{array}$ & $\begin{array}{c}\text { Actual } \\
\text { value }\end{array}$ & $\begin{array}{c}\text { Predicted } \\
\text { value }\end{array}$ & $\begin{array}{l}\mathrm{S} / \mathrm{N} \\
\text { ratio }\end{array}$ \\
\hline 1 & PLX-188 & D-114 & 150 & 100 & 10 & $1.5: 0.5$ & $1.5: 0.5$ & 180 & 105.3 & 107.91667 & -47.9 & 81.7 & 84.77778 & -35.34 & 8.8 & 8.7666667 & -25.43 \\
\hline 2 & PVA & D-118 & 200 & 100 & 30 & $1.0: 1.0$ & $0.5: 1.5$ & 180 & 182.9 & 182.93333 & -45.4 & 78.6 & 77.45556 & -43.12 & 8.9 & 8.05 & -19.7 \\
\hline 3 & PLX-188 & D-114 & 100 & 200 & 30 & $1.0: 1.0$ & $1.0: 1.0$ & 120 & 207.1 & 201.25 & -40.64 & 83.1 & 84.77778 & -36.12 & 7.7 & 8.76666667 & -20.9 \\
\hline 4 & PVA & D-116 & 100 & 100 & 20 & $1.0: 1.0$ & 1.5:0.5 & 240 & 126.9 & 131.18333 & -43.01 & 76.7 & 77.45556 & -37.36 & 8.6 & 8.8833333 & -22.33 \\
\hline 5 & PVA & D-114 & 100 & 100 & 10 & $0.5: 1.5$ & $0.5: 1.5$ & 120 & 122.9 & 126.23333 & -46.88 & 76.1 & 77.45556 & -35.13 & 6.8 & 6.7 & -20.78 \\
\hline 6 & PLX-188 & D-118 & 150 & 100 & 30 & $0.5: 1.5$ & $1.0: 1.0$ & 240 & 164.5 & 166.26667 & -42.55 & 81.5 & 84.77778 & -38.73 & 8.5 & 8.8833333 & -22.08 \\
\hline 7 & PVA & D-118 & 150 & 200 & 20 & $0.5: 1.5$ & $1.5: 0.5$ & 120 & 178.9 & 166.16667 & -45.32 & 76.5 & 77.45556 & -37.97 & 7.8 & 8.05 & -23.35 \\
\hline 8 & PLX-188 & D-118 & 200 & 100 & 10 & $1.0: 1.0$ & $1.5: 0.5$ & 120 & 122.8 & 126.23333 & -42.46 & 85.4 & 84.77778 & -36.78 & 11.2 & 10.116667 & -23.77 \\
\hline 9 & PLX-188 & D-118 & 100 & 150 & 20 & $1.5: 0.5$ & $0.5: 1.5$ & 180 & 160.9 & 147.85 & -51.29 & 85.9 & 84.77778 & -35.89 & 10.4 & 10.116667 & -20.64 \\
\hline 10 & PVA & D-118 & 100 & 150 & 10 & $1.5: 0.5$ & $1.0: 1.0$ & 240 & 98.3 & 91.25 & -44.63 & 78.2 & 77.45556 & -34.76 & 7.7 & 8.05 & -19.85 \\
\hline 11 & PVA & D-116 & 150 & 150 & 30 & $1.5: 0.5$ & $0.5: 1.5$ & 120 & 201.7 & 201.25 & -38.89 & 78.9 & 77.45556 & -31.64 & 8.2 & 8.8833333 & -29.16 \\
\hline 12 & PVA & D-114 & 200 & 200 & 30 & $1.5: 0.5$ & $1.5: 0.5$ & 240 & 162.4 & 166.26667 & -36.97 & 76.1 & 77.45556 & -37.26 & 7.2 & 6.7 & -24.98 \\
\hline 13 & PLX-188 & D-116 & 100 & 150 & 30 & $0.5: 1.5$ & $1.5: 0.5$ & 180 & 182.3 & 182.93333 & -45.22 & 86.1 & 84.77778 & -35.13 & 10.7 & 10.95 & -25.76 \\
\hline 14 & PLX-188 & D-116 & 200 & 100 & 20 & $1.5: 0.5$ & $1.0: 1.0$ & 120 & 153.9 & 166.16667 & -38.08 & 86.2 & 84.77778 & -32.65 & 12.1 & 10.95 & 23.86 \\
\hline 15 & PLX-188 & D-116 & 150 & 200 & 10 & $1.0: 1.0$ & $0.5: 1.5$ & 240 & 94.6 & 91.25 & -45.25 & 87.4 & 84.77778 & -35.53 & 11.4 & 10.95 & -18.13 \\
\hline 16 & PLX-188 & D-114 & 200 & 150 & 20 & $0.5: 1.5$ & $0.5: 1.5$ & 240 & 130.7 & 131.18333 & -39.55 & 85.7 & 84.77778 & -37.01 & 8.7 & 8.76666667 & -23.16 \\
\hline 17 & PVA & D-114 & 150 & 150 & 20 & $1.0: 1.0$ & $1.0: 1.0$ & 180 & 139.1 & 147.85 & -44.36 & 77.7 & 77.45556 & -31.36 & 7.2 & 6.7 & -16.15 \\
\hline 18 & PVA & D-116 & 200 & 200 & 10 & $0.5: 1.5$ & $1.0: 1.0$ & 180 & 106.9 & 107.91667 & -42.89 & 78.3 & 77.45556 & -40.06 & 8.5 & 10.116667 & -17.43 \\
\hline
\end{tabular}

Table 2: The mean particle size, PDI, zeta potential, entrapment efficiency and \% drug loading of optimized formulations

\begin{tabular}{cccccc}
\hline Batch & $\begin{array}{c}\text { MPS } \pm \text { SD } \\
(\mathbf{n m})\end{array}$ & PDI & $\begin{array}{c}\text { ZP } \pm \text { SD } \\
(\mathbf{m V})\end{array}$ & $\begin{array}{c}\text { \% EE } \pm \\
\text { SD }\end{array}$ & $\begin{array}{c}\text { \% DL } \pm \\
\text { SD }\end{array}$ \\
\hline 1 & $95.57 \pm 6.3$ & 0.283 & $-31.8 \pm 3.15$ & 91.24 & 11.92 \\
2 & $91.34 \pm 5.2$ & 0.247 & $-29.3 \pm 4.89$ & 90.63 & 12.32 \\
3 & $94.14 \pm 6.8$ & 0.240 & $-28.2 \pm 5.14$ & 90.78 & 12.16 \\
\hline $\mathrm{n}=3(p<0.05)$ & & & &
\end{tabular}

Table 3: Dissolution profile of Tazarotene solid lipid nanoparticles (Optimized batches)

\begin{tabular}{ccccc}
\hline Time & \multicolumn{5}{c}{ \% CDR } \\
\cline { 2 - 5 }$($ min) & Pure drug & F1 & F2 & F3 \\
\hline 0 & 0 & $0 \pm 0$ & $0 \pm 0$ & $0 \pm 0$ \\
5 & 5.34 & $11.8 \pm 1.28$ & $12.19 \pm 2.62$ & $12.31 \pm 1.72$ \\
10 & 9.36 & $26.05 \pm 1.52$ & $27.94 \pm 1.12$ & $27.87 \pm 0.76$ \\
15 & 13.56 & $42.30 \pm 1.25$ & $42.41 \pm 1.34$ & $41.92 \pm 2.01$ \\
20 & 18.93 & $59.40 \pm 1.19$ & $58.92 \pm 0.96$ & $59.12 \pm 1.32$ \\
30 & 27.72 & $71.50 \pm 1.27$ & $70.83 \pm 1.56$ & $71.46 \pm 1.65$ \\
40 & 32.98 & $80.76 \pm 1.28$ & $79.93 \pm 2.21$ & $81.02 \pm 0.64$ \\
50 & 37.43 & $89.27 \pm 1.58$ & $88.93 \pm 1.35$ & $89.42 \pm 1.89$ \\
60 & 42.12 & $98.12 \pm 1.52$ & $97.63 \pm 0.97$ & $97.91 \pm 0.62$ \\
\hline
\end{tabular}

Table 4: Flux of Tazarotene from SLN based gel formulation

\begin{tabular}{ccc}
\hline \multirow{2}{*}{ Formulation } & Flux $\left(\mathbf{\mu g ~ \mathbf { ~ m } ^ { - 2 } \mathbf { h }} \mathbf{- 1}\right)$ & \\
\cline { 2 - 3 } & Egg membrane & Rat skin \\
\hline F1 & $54.354 \pm 1.43$ & $193.454 \pm 4.324$ \\
Marketed formulation & $35.366 \pm 4.12$ & $116.345 \pm 2.238$ \\
Pure drug & $26.114 \pm 1.89$ & $39.654 \pm 3.12$ \\
\hline
\end{tabular}

Table 5: Mean erythemal scores for various Tazarotene formulations obtained at the end of 24,48 and $72 \mathrm{~h}$

\begin{tabular}{|c|c|c|c|}
\hline \multirow{2}{*}{ Formulations } & \multicolumn{3}{|c|}{ Erythmeal score $(n=3)$} \\
\hline & $24 \mathrm{~h}$ & $48 \mathrm{~h}$ & $72 \mathrm{~h}$ \\
\hline Pure drug (Group 1) & 0 & 0 & 0 \\
\hline Marketed gel (Group 2) & 1 & 1 & 1 \\
\hline $\begin{array}{l}\text { SLN based gel without Tazarotene } \\
\text { (Group 3) }\end{array}$ & 0 & 0 & 0 \\
\hline $\begin{array}{l}\text { SLN based gel containing Tazarotene } \\
\text { (Group 4) }\end{array}$ & 0 & 0 & 0 \\
\hline
\end{tabular}

The mean size of all the formulations was ranging from $91.34 \pm 5.2 \mathrm{~nm}$ to $95.57 \pm 6.3 \mathrm{~nm}$ The PDI was ranging from 0.240 to 0.283 , indicating the narrow size distribution. The SLN formulations exhibited negative surface charge with the inclusion of Tazarotene which clearly suggested the orientation of in the lipid matrix. The zeta potential values of SLN formulations were found to be in between to $-28.2 \pm 5.14 \mathrm{mV}$ to $-31.8 \pm 3.15$
$\mathrm{mV}$. Total entrapment efficiency of the nanoparticles formulations was determined and found to be ranging from $90.63 \%$ to $91.24 \%$. The percent drug loading of the formulations was found to be in the range from $11.92 \%$ to $12.32 \%$ (Table 2 ).

\section{Drug release study}

Dissolution rates of Tazarotene pure drug suspension and solid lipid nanoformulations were evaluated. All formulations showed an increase in dissolution over pure drug, which showed only $\approx 42 \%$ release after 60 min. The relatively higher dissolution enhancement could be credited to more intimate drug carrier interaction during formulation of nanoparticles. Based on the data formulation F1 is considered for further investigation. The in vitro drug release pattern of drug from the optimized batches is as shown in Table 3 and Figure 1.

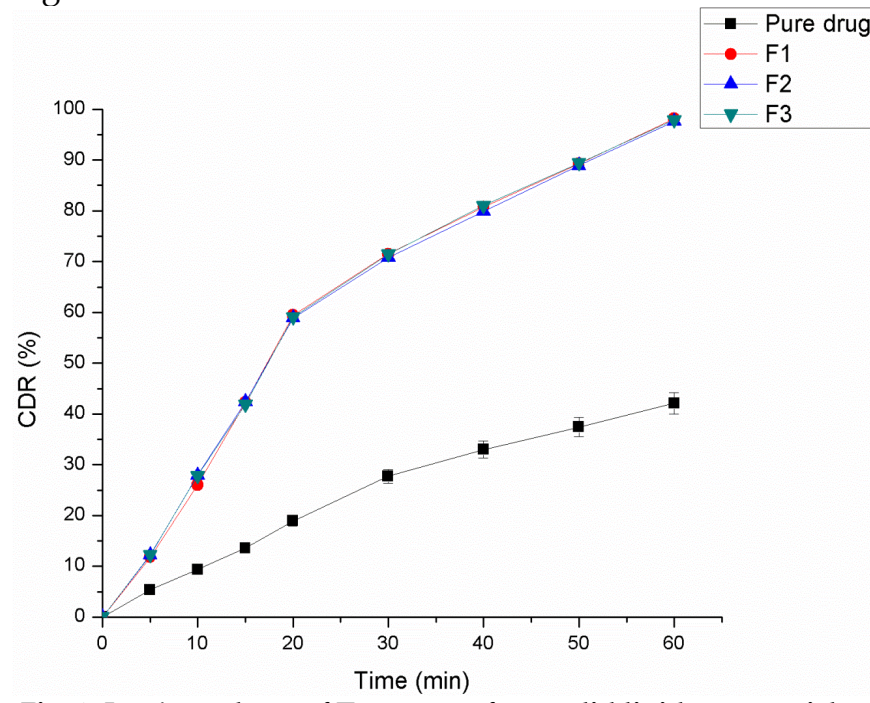

Fig. 1: In-vitro release of Tazarotene from solid lipid nanoparticles

\section{Evaluation of SLN based gel of Tazarotene}

The Tazarotene content of the gel formulation was found to $98.96 \pm 0.021 \% \mathrm{w} / \mathrm{w}$ of the theoretical value $(0.05 \% \mathrm{w} / \mathrm{w})$. The results indicate that the processes employed to prepare solid dispersions in this study were capable of producing formulation with uniform drug content. 
The diameter of gel formulation was $6.76 \pm 0.021 \mathrm{~cm}$ and that of marketed gel was found to be $4.9 \pm 0.089$ $\mathrm{cm}$, indicating better spreadability of SLN based gel formulation. This is because of the loose gel matrix nature of gel formulation due to the presence of oil globules rather than the conventional gel matrix.

The $\mathrm{pH}$ of the gel was found to be $6.8 \pm 0.06$ indicating that it could result in less irritation to the skin. The viscosity of gel formulation at $5 \mathrm{rpm}$ was found to be $5.98 \times 10^{3} \pm 0.34 \times 10^{3} \mathrm{cp}$.

The release rate (flux) of Tazarotene across the membrane and excised skin differs significantly (Table 4), which indicates about the barrier properties of skin. The flux value for SLN based gel formulation (193.454 \pm $4.324 \mu \mathrm{g} \mathrm{cm}^{-2} \mathrm{~h}^{-1}$ ) was found to be higher than that for marketed gel $\left(116.345 \pm 2.238 \mu \mathrm{g} \mathrm{cm}^{-2} \mathrm{~h}^{-1}\right)$. The higher flux and Kp values of SLN based gel suggest that it might be able to enter the skin easily as compared with marketed gel with an advantage of low interfacial tension of the emulsifier film that ensures an excellent contact to the skin.

The accumulative amount of Tazarotene in skin from SLN based gel formulation and marketed gel were $41.12 \pm 0.12 \mathrm{mg}$ and $30.02 \pm 0.04 \mathrm{mg}$ respectively. This result supported our hypothesis made in skin permeation studies on rat skin. The more tazarotene permeates the less is retained in the skin and might lead to systemic adverse side effects. This was one of the reasons to employ lipid based formulations for topical delivery of Tazarotene as its epidermal localization is highly desirable for enhancing the treatment of skin diseases such as psoriasis.

A
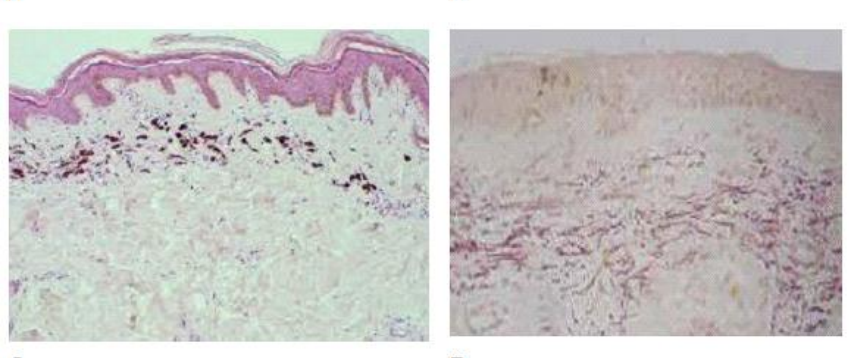

C

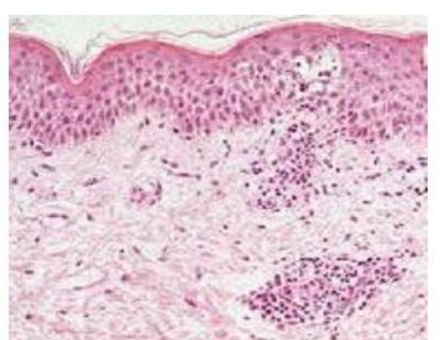

D

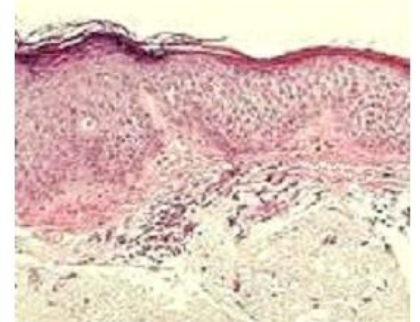

Fig. 2: Light microscopic photographs of in vitro histological study. (A) Pure drug; (B)The rat skin treated with optimized SLN formulation; (C)The rat skin treated with SLN based gel formulation; (D)The rat skin treated with marketed gel.

The rat skin is a multilayered organ with many histological layers. The histology of excised rat skin in pure drug and treated with optimized Tazarotene SLN formulation, Tazarotene SLN gel formulation and marketed gel is shown in Figure 2 (A-D). The microscopic observations indicate that the optimized SLN formulation, SLN based gel formulation and marketed gel has no significant effect on the microscopic structure of the skin. The surface epithelium lining and the granular cellular structure of the skin were totally intact. No major changes in the ultra structure of skin morphology could be seen and the epithelial cells appeared mostly unchanged.

One of the major disadvantages associated with the Tazarotene therapy is skin irritation (erythema), which strongly limits its utility and acceptability by the patients. Ideally, the delivery system of Tazarotene should be able to diminish or abolish these erythematic episodes. However, most of the currently marketed conventional dosage forms such as creams, lotions and gels are not able to reduce the irritation caused by topical application of Tazarotene. It was hypothesized that encapsulation of Tazarotene in the lipid structure would reduce the skin irritation (Table 5).

Draize patch test is a reliable method and the results obtained from this study can be linked to that obtained in humans. The skin-irritation studies indicated that SLN based gel containing Tazarotene did not show any sign of skin irritation as compared to moderate erythema shown by marketed gel formulation (Tazret $\AA$ gel) after $72 \mathrm{~h}$ of application (Table 5). Thus, SLN based gel formulation demonstrated advantage over marketed formulation in improving the skin tolerability of Tazarotene indicating their potential in improving patient acceptance and topical delivery of Tazarotene.

The results of the research work could be concluded as successful production of Tazarotene SLN's by the ultrasonication using Taguchi's design, and based on the results further investigation was made using central composite design. The optimized formulation was incorporated into the gel. The accumulative amount of Tazarotene in skin from SLN based gel formulation and marketed gel were $41.12 \pm 0.12 \mathrm{mg}$ and $30.02 \pm 0.04 \mathrm{mg}$ respectively. This result supported our hypothesis made in skin permeation studies on rat skin. From histopathological studies the microscopic observations indicate that the optimized SLN formulation, SLN based gel formulation and marketed gel has no significant effect on the microscopic structure of the skin The skin-irritation studies indicated that SLN based gel containing Tazarotene did not show any sign of skin irritation as compared to moderate erythema shown by marketed gel formulation after $72 \mathrm{~h}$ of application. Thus, SLN based gel formulation demonstrated advantage over marketed formulation in improving the skin tolerability of Tazarotene indicating their potential in improving patient acceptance and topical delivery of Tazarotene.

\section{REFERENCES}

1. Meghana G, Kari VVSNR, Siddhartha VN, Dwija T, Ganesh GNK. In vitro and in vivo behavior of a carbamothioic acid liposomal gel for the treatment of topical fungal diseases. Int J Chem Tech Res. 2015; 7:814-20. 
2. Dubey A, Kamath J. Nanostructured lipid carriers: a novel topical drug delivery system. Int J Pharm Tech Res. 2012; 4: 705-14.

3. Hadgraft J. Passive enhancement strategies in topical and transdermal drug delivery. Int J Pharm. 1999; 184: 1-6.

4. Kreilgaard M. Influence of microemulsions on cutaneous drug delivery. Adv Drug Delivery Rev. 2002; 54: 77-98.

5. Müller R, Radtke M, Wissing S. Solid lipid nanoparticles (SLN) and nanostructured lipid carriers (NLC) in cosmetic and dermatological preparations. Adv Drug Delivery Rev. 2002; 54:131-55.

6. Muller-Goymann CC. Physiochemical characterization of colloidal drug delivery systems such as reverse micelles, vesicles, liquid crystals and nanoparticles for topical administration. Eur J Pharm Biopharm. 2004; 58: 343-56.

7. Manjunath K, Venkateswarlu V. Pharmacokinetics, tissue distribution and bioavailability of clozapine solid lipid nanoparticles after intravenous and intraduodenal administration. J Contr Rel. 2005; 107: 215-28.

8. Muller RH, Mader K, Gohla S. Solid lipid nanoparticles (SLN) for controlled drug delivery a review of the state of the art. Eur J Pharm Biopharm. 2000; 50:161-77

9. Cavalli R, Caputo O, Carlotti ME. Sterilization and freeze drying of drug-free and drug-loaded solid lipid nanoparticles. Int J Pharm. 1997; 148:47-54

10. Emami J, Mohiti H, Hamishehkar H, Varshosaz J. Formulation and optimization of solid lipid nanoparticle formulation for pulmonary delivery of budesonide using Taguchi and Box-Behnken design. Res Pharm Sci. 2015; 10(1) 17-33.
11. Roy A. Primer on the Taguchi Method. Van Nostrand Reinhold, New York, 1990

12. Maia CS, Mehnert W, Schafer-Korting M. Solid lipid nanoparticles as drug carriers for topical glucocorticoids. Int J Pharm. 2000; 196:165-7.

13. Bachhav YG, Patravale VB. Microemulsion based vaginal gel of fluconazole: Formulation, in vitro and in vivo evaluation. Int J Pharm. 2009; 365:175-179.

14. Laithy HM, Shaboury KMF. The development of cutinalipogels and gel microemulsion for topical administration of fluconazole. AAPS Pharm Sei Tech. 2002; 3 : article 35.

15. Mehnert W, Mader K. Solid lipid nanoparticles: production, characterization and applications. Advanced Drug Delivery Reviews 2012; 64:83-101.

16. 16. Patel MR, Patel RB, Parikh JR. Effect of formulation components on the in vitro permeation of microemulsion drug delivery system of fluconazole. AAPS Pharm Sci Tech. 2009; 10:917-923.

17. Biruss B, Valenta C. The advantage of polymer addition to a non-ionic oil in water microemulsion for the dermal delivery of progesterone. Int J Pharm. 2008; 349: 269 -273.

18. Patel RB, Patel MR, Bhatt KK, Patel BG. Risperidone loaded mucoadhesive microemulsion for intranasal delivery: formulation, development, physicochemical characterization and ex vivo evaluation. J Drug Del Sci Tech. 2013; 23: 261267.

19. Draize J, Woodard G, Calvery H. Methods for the study of irritation and toxicity of substances topically applied to skin and mucous membranes. J Pharmacol Exp Ther. 1944; 82: 377-390. 